\title{
Control of Incoming Drain Currents Drawn by Super-Junction MOSFETs in Voltage Source Bridge-Legs
}

\author{
Zhengyang Feng \\ Department of Electronic \& Electrical \\ Engineering \\ University of Strathclyde \\ Glasgow, UK \\ zhengyang.feng@strath.ac.uk
}

\author{
Neville McNeill \\ Department of Electronic \& Electrical \\ Engineering \\ University of Strathclyde \\ Glasgow, UK \\ neville.mcneill@strath.ac.uk
}

\author{
Barry Williams \\ Department of Electronic \& Electrical \\ Engineering \\ University of Strathclyde \\ Glasgow, UK \\ barry.williams@strath.ac.uk
}

\begin{abstract}
To increase the efficiency of renewable energy power conversion systems, traditional silicon IGBTs can be replaced with silicon super-junction MOSFETs. However, the poor performance of the MOSFET's intrinsic diode and the output capacitance present difficulties in voltage source converter bridge-legs. When a MOSFET in this circuit turns on, a charging current has to be sourced into the output capacitance of the complementary freewheeling MOSFET, even if the diode has been deactivated. The peak incoming drain current into the MOSFET turning on can be limited by using a large resistance in series with its gate. However, this increases MOSFET power dissipation. Also, the turn-on propagation delay time is increased. This paper presents a gate driver circuit for profiling the MOSFET's incoming drain current to provide an improved trade-off between incoming peak current, turn-on power dissipation, and delay time.
\end{abstract}

Keywords - control, converter, current transformer, gate driver, MOSFET, super-junction.

\section{INTRODUCTION}

High power conversion efficiencies are desirable in many renewable energy applications. The traditional silicon IGBT can be replaced with wide band-gap (WBG) devices including silicon carbide ( $\mathrm{SiC}$ ) MOSFETs, SiC JFETs, and GaN HEMTs. WBG devices [1] offer excellent performance. However, they present challenges. For example, $\mathrm{d} v / \mathrm{d} t$ induced conduction and gate threshold shift can be problems with the SiC MOSFET [2].

Another alternative to the IGBT is the silicon superjunction (SJ) MOSFET. This device has a low on-state resistance $R_{D S(o n)}$, is fast-switching and exhibits good shortcircuit withstand times [3]. The SJ MOSFET is effective in single-ended converters such as the boost converter when used in conjunction with a SiC Schottky diode [4]. However, the voltage source converter (VSC) bridge-leg is a key element in many renewable power systems, such as with wind turbine generators. In VSCs the high reverse recovery charge $Q_{r r}$ passed by the Si SJ MOSFET's intrinsic diode, and its highly non-linear output capacitance $C_{\text {oss }}$ [5] present challenges.

Solutions include the use of auxiliary bridge-legs [6], switching-aid circuits [7], and intelligent commutation schemes such as those in [8] and [9]. SJ MOSFETs can be deployed in a VSC operating in the synchronous conduction mode. However, a variable switching frequency is used, so $\mathrm{AC}$ filter design is challenging. The functionality of a VSC can be realized by combining single-ended converters in, for example, the dual-buck arrangement in [10] but factors such as choke utilization can be problematic. As an alternative to the VSC, the SJ MOSFET has been shown to perform effectively in both the current source converter (CSC) [11], and the impedance source converter (ZSC) [12]. However, challenges with the CSC include the need to invert the DC voltage if the power flow is to be reversed. The ZSC requires additional components to form the required impedance network. For these and other reasons, the VSC tends to be prevalent in practical power systems.

\section{BACKGROUnd: DuAL-Mode CONTROL TECHNIQUe}

\section{A. Operating Principle}

Fig. 1(a) shows a bridge-leg with MOSFET intrinsic diode deactivation circuitry incorporated. When a MOSFET would be freewheeling, the reverse current, rather than flowing into its intrinsic diode, will flow into the parallel external diode because of the blocking action of the series diode. The external diodes can be purpose-designed types with good reverse recovery characteristics, such as $\mathrm{SiC}$ Schottky types. However, the highly non-linear output capacitance $C_{\text {oss }}$ of the MOSFET in the freewheeling position is still problematic.

[13] proposed using a dual-mode control technique in conjunction with the circuitry in Fig. 1(a) to address the remaining problem presented by $C_{\text {oss }}$, shown in Fig. 2(a). In the so-called fast-switching mode, the bridge-leg operates with unipolar PWM modulation according to the direction of the AC load current. Under unipolar PWM modulation, the MOSFET in the freewheeling location is not turned on during a switching cycle. Consequently, its $C_{\text {oss }}$ is only partially discharged. This is shown in Fig. 1(b), where $C_{o s s}$ discharges down to the reverse breakdown voltage of $D_{s}$ which is appropriately chosen to be above the voltage of the knee point of the $Q-V$ curve in Fig. 2(a). In this mode, TR 1 and $T R 2$ do not have to dissipate the full co-energy lying under the curve in Fig. 2(a) in the course of charging the $C_{o s s}$ of the complementary device. $T R 1$ and $T R 2$ are driven with rapid switching for low losses.

At low $i_{L O A D}$ magnitudes, bipolar PWM modulation is enabled to reduce crossover distortion in the AC current. The problem of high peak currents due to supplying high charges into fully-discharged $C_{o s s}$ capacitances that occurs with bipolar modulation, is addressed by turning the SJ MOSFETs on slowly (the so-called slow-switching mode) and thereby limiting the $\mathrm{d} i / \mathrm{d} t$ in the current conducted by the incoming MOSFET as shown in Fig. 2(b). Fig. 3 shows simulation results for the dual-mode technique over one base-frequency cycle of $i_{L O A D}$, where the fast-switching and slow-switching modes are selected according to the magnitude of $i_{L O A D}$. The 


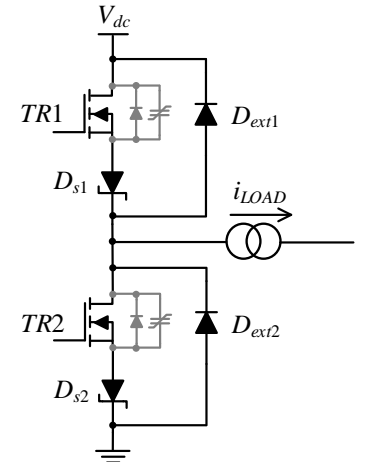

(a) (b)

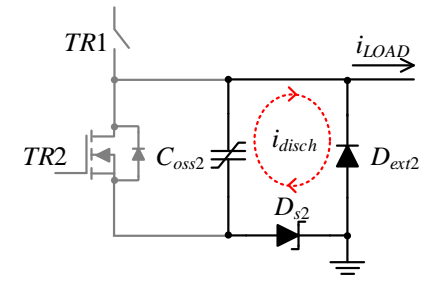

Fig. 1 (a) Voltage source bridge-leg based around SJ MOSFETs equipped with intrinsic diode deactivation circuitry. (b) Route taken by discharge current from output capacitance $C_{o s s 2}$ when $T R 1$ is off and TR2 is freewheeling.

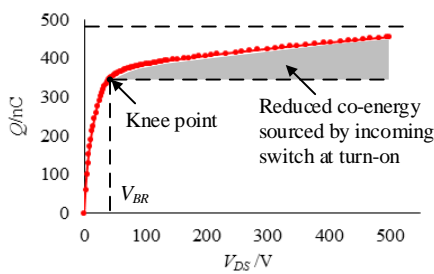

(a)

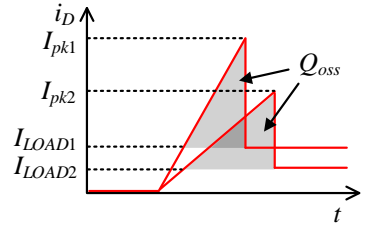

(b)
Fig. 2 (a) $Q-V$ curve of IPW60R041P6 SJ MOSFET's $C_{\text {oss }}$ (b) Same $Q_{\text {oss }}$ under different $I_{L O A D}$ and different slew rate where load current $I_{L O A D 1}>I_{L O A D 2}$, peak current $I_{p k 1}>I_{p k 2}$.

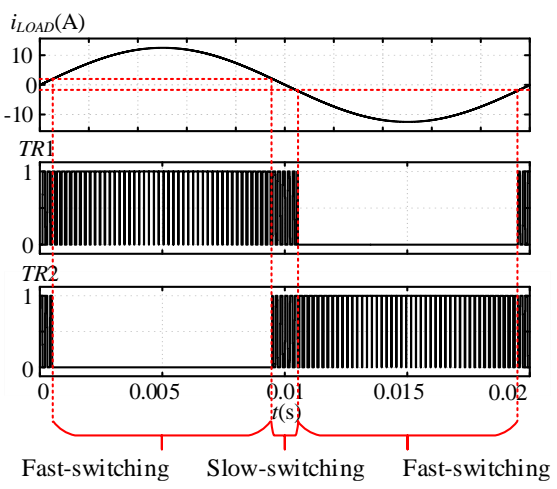

Fig. 3 Simulation results of dual-mode control technique.

dual-mode technique was demonstrated in an 800-W inverterleg configuration operating from a 400-V DC supply voltage, and switching at $20 \mathrm{kHz}$ in [13]. The full-load efficiency was $98.7 \%$, compared with $97.5 \%$ measured with a benchmark circuit using IGBTs. A feature of the technique is that compared to, for example, [6]-[9], little extra hardware is required. Also, the SJ MOSFETs do not have to support overvoltages during the resetting action of switching-aid inductors, as in [7].

With respect to Fig. 1(a), DeWitt et al. proposed a technique in [14] where the series diodes are replaced with low breakdown voltage MOSFETs. This reduces the conduction losses of the series elements as the MOSFETs act as synchronous rectifiers, with smaller voltage drops than series diodes. However, as with the basic circuitry in Fig. 1(a), this still does not address the problem of the non-linear MOSFET output capacitances.

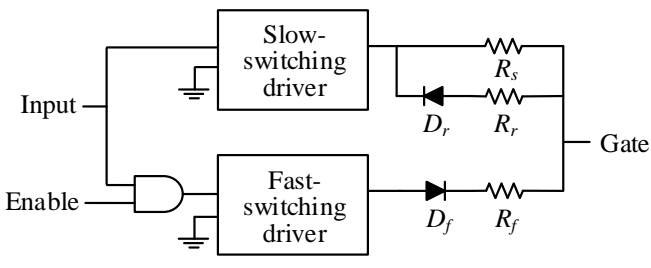

Fig. 4 Dual-mode gate driver circuit from [13].

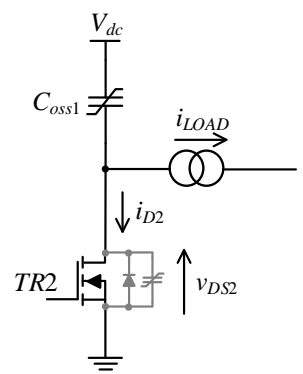

Fig. 5 Equivalent circuit from bridge-leg in Fig. 1(a) when $i_{L O A D}$ is negative and $T R 2$ is turning on, and the intrinsic diode in $T R 1$ has been deactivated.

\section{B. Dual-Mode Gate Driver Hardware}

Fig. 4 shows the dual-mode gate driver circuit. When the slow-switching mode is selected, only the slow-switching driver operates and the MOSFET's input capacitance $C_{i s s}$ is charged at turn-on via the relatively high-value resistance $R_{s}$. When the fast-switching driver is enabled, $C_{i s s}$ is charged more rapidly via the parallel combination of $R_{s}$, and the much smaller resistance $R_{f}$.

\section{Issues with Incoming Current Profile in Slow-Switching Mode}

In Fig. 5, the turn-on action of TR2 is considered when the bridge-leg has $i_{L O A D}$ flowing into its mid-point. $i_{L O A D}$ is taken as being low, and hence the converter is operating in the slowswitching mode. If the intrinsic diode of $T R 1$ has been deactivated effectively, $T R 1$ 's equivalent circuit can be approximated solely by its output capacitance $C_{o s s 1}$. The reverse recovery charge of $D_{\text {ext } 2}$ is low in comparison with that drawn by $C_{o s s}$, so $D_{e x t 2}$ is neglected here.

In Fig. 5, the peak incoming drain current $i_{D 2}$ is limited by using a large gate resistance. A feature of this arrangement is the inherently triangular incoming drain current profile with respect to time, as shown in Fig. 6. When the gate-source voltage $v_{G S}$ reaches its threshold level $V_{G S(t h)}$ at Point A, the MOSFET has the full supply voltage $V_{d c}$ across its drainsource terminals. It then begins to conduct whilst in the pinchoff condition, and still supports virtually all of $V_{d c}$. $i_{D 2}$ rises approximately linearly until the bulk of the total charge $Q_{o s s}$ has been supplied into $C_{o s s}$ at Point B. During this phase $v_{G S}$ has increased in an approximately linear manner with respect to time as the change in $v_{G S}$ has been over only a small section of an exponential curve. At Point B, $C_{o s s 1}$ falls abruptly and, in moving to Point $\mathrm{C}, i_{D 2}$ falls to the steady-state load current level and $v_{D S 2}$ falls from the rail voltage to virtually zero.

A disadvantage of the triangular current profile is that the overshoot current $I_{o s}$, as defined in Fig. 6(c), is high. When $C_{o s s}$ falls, a high current has been established in both $T R 1$ and $T R 2$. Interrupting this current can result in high-frequency oscillations in the voltage at the source of $T R 1$. This, in turn, can result in a transient voltage exceeding $V_{d c}$ appearing across $T R 1$, and an EMI signature with a raised high-frequency 
content. $I_{o s}$ can be reduced by switching more slowly to address these problems shown in Fig. 2(b). However, this incurs increased losses in the MOSFET as it spends more time with the rail voltage across it whilst conducting both $i_{L O A D}$ and the $C_{\text {oss }}$-charging current.

Using a large gate resistance to reduce the turn-on speed of a MOSFET has a further disadvantage as the time delay $D$ between the gate signal from the driver IC going high and the MOSFET's gate voltage reaching its threshold value becomes lengthened. Distortion results in the output voltage and current due to this propagation delay between the MOSFET being signalled on and its drain-source voltage falling. by

With respect to the waveforms in Fig. 6(c), $Q_{\text {oss }}$ is given

$$
Q_{o s s}=1 / 2 t_{2} I_{o s} .
$$

(1) is rearranged to give

$$
t_{2}=\frac{2 Q_{o s s}}{I_{o s}} .
$$

The rate of rising of $i_{D 2}$ is taken as being the same during the intervals $t_{1}$ and $t_{2}$, and therefore

$$
\frac{I_{L O A D}}{t_{1}}=\frac{I_{o s}}{t_{2}} .
$$

(3) is rearranged to give

$$
t_{1}=\frac{I_{L O A D}}{I_{o s}} t_{2}
$$

Putting the result from (2) into (4) gives

$$
t_{1}=\frac{2 I_{L O A D} Q_{o S S}}{I_{o s}{ }^{2}} \text {. }
$$

The results from (2) and (5) are added to give the total time $T_{T O T A L}$ for $i_{D 2}$ to rise from 0 A to $I_{L O A D}+I_{o s}$ :

$$
T_{T O T A L}=\frac{2 Q_{\text {OSS }}}{I_{O S}}\left(\frac{I_{L O A D}}{I_{O S}}+1\right) .
$$

The energy dissipation $E_{\text {on(tri) }}$ in $T R 2$ with a triangular incoming drain current trajectory is given by

$$
E_{\text {on(tri) }}=V_{d c}\left(\frac{I_{L O A D}+I_{o s}}{2}\right) T_{T O T A L} .
$$

Putting the result from (6) into (7), and rearranging yields

$$
E_{\text {on(tri) }}=V_{d c} Q_{o s s}\left(1+\frac{2 I_{L O A D}}{I_{o s}}+\frac{I_{L O A D}{ }^{2}}{I_{o s}{ }^{2}}\right) \text {. }
$$

Compared to the triangular profile, a rectangular profile, Fig. 7, would be expected to yield lower turn-on losses for a given overshoot current. With respect to the waveforms in Fig. 7, $Q_{o s s}$ is given by

$$
Q_{o s s}=t_{1} I_{o s}
$$

(9) is rearranged to give

$$
t_{1}=\frac{Q_{o s s}}{I_{o s}} .
$$

The energy dissipation $E_{\text {on(rec })}$ in $T R 2$ with a rectangular incoming drain current trajectory is given by

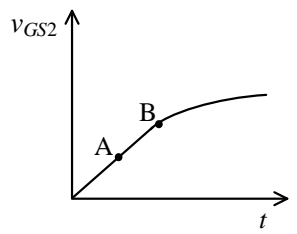

(a)

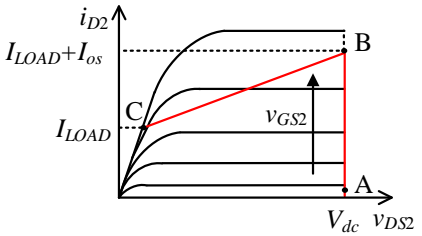

(b)

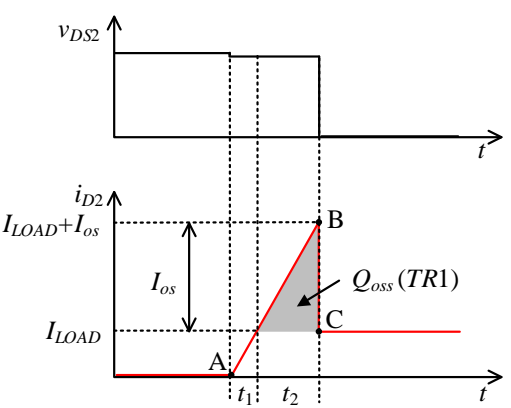

(c)

Fig. 6 Idealized behavior of SJ MOSFET in bridge-leg at TR2 turn-on, showing its incoming drain current profile. (a) $v_{G S 2}$ as a function of time. (b) $i_{D 2}$ as a function of $v_{D S 2}$. (c) $v_{D S 2}$ as a function of time, $i_{D 2}$ as a function of time.

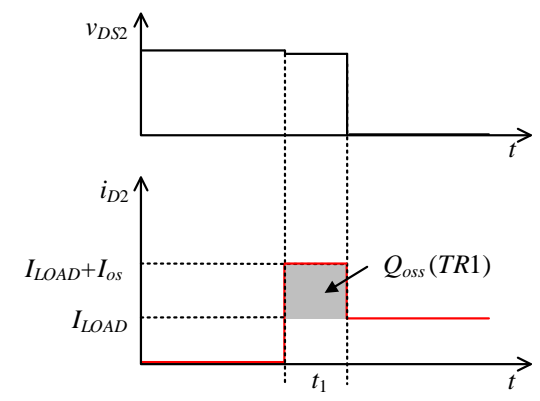

Fig. 7 Idealized rectangular incoming drain current profile.

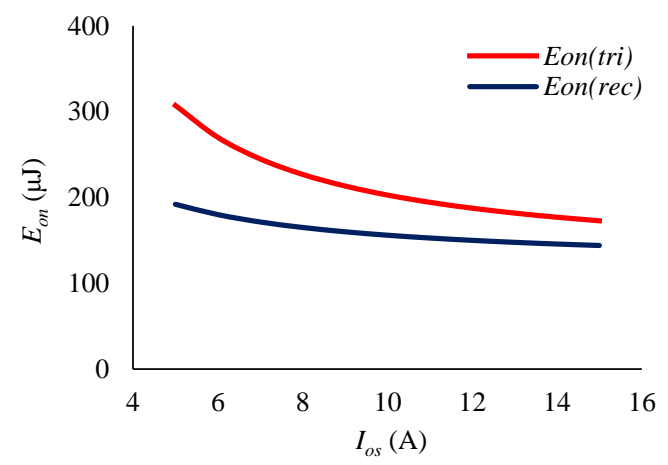

Fig. 8 Expected turn-on energies $E_{o n(t r i)}$ and $E_{o n(r e c)}$ shown against $I_{o s}$.

$$
E_{\text {on(rec) }}=V_{d c}\left(I_{L O A D}+I_{o s}\right) t_{1} .
$$

The result from (10) is put into (11) to yield

$$
E_{\text {on(rec })}=V_{d c} Q_{o s s}\left(1+\frac{I_{L O A D}}{I_{o s}}\right) \text {. }
$$

Fig. 8 shows $E_{\text {on(tri) }}$ and $E_{\text {on(rec })}$ plotted against overshoot current $I_{o s}$ for triangular and rectangular trajectories for some representative conditions. These were $V_{d c}=400 \mathrm{~V}, I_{L O A D}=$ $3 \mathrm{~A}$, and $Q_{o s s}=300 \mathrm{nC}$. (8) and (12) were used to calculate the turn-on energies. 


\section{Proposed Circuitry for Realizing a Rectangular INCOMING DRAIN CURRENT PROFILE}

A technique was presented in [15] for controlling the incoming diode recovery current profile drawn by a MOSFET in a single-ended converter. As shown in Fig. 9, a current transformer (CT) is used to provide a diode recovery current control arrangement. When $Q_{1}$ turns on, and the load current is commutated from $D_{1}$ to $Q_{1}$, a voltage drop across $R_{2}$ is developed as a function of the current in the primary conductor of the CT. With an appropriately chosen $R_{2}$ and CT turns-ratio, the drain current of $Q_{1}$ will be limited to a set value during the diode recovery period.

In this paper, the technique in [15] is extended to a VSC bridge-leg where the incoming drain currents in two SJ MOSFETs need to be controlled. Also, the resetting of the CT is addressed; the technique is applied in conjunction with a dual-mode gate driver arrangement; its efficacy is experimentally compared with that of a traditional arrangement where the incoming current profile is determined by simply setting the gate resistance to a desired value. A feature of the arrangement in [15] is that the CT is used to both detect the recovery current and control it. Other techniques can be used for sensing currents in power devices, for protection against short-circuits or to obtain the current profile for active shaping of currents during switching transients. Methods include the use of a Rogowski coil [16] and detection of the voltage developed across the parasitic emitter inductance of an IGBT [17], [18]. The advantage of the CT here is that its output can be used to directly control the MOSFET's drain current, without the need for integrators or other conditioning circuitry.

Figs. 10 and 11 show the proposed circuitry. In Fig. 10 it is seen that the primary winding of each CT is connected directly in series with each MOSFET. As shown in Fig. 11, the CT in each case acts to limit the gate voltage. This differs from [15] where the primary conductor of the CT limiting the current flow into a MOSFET was connected in series with the freewheeling element sourcing the incoming recovery current. It is noted that the current being directly controlled with the proposed arrangement is therefore not the reverse current through the freewheeling element, as in [15], but is the peak current $I_{p k}\left(I_{L O A D}+I_{o s}\right)$ in the incoming MOSFET. As shown in Fig. 7, $I_{p k}$ is composed of both $i_{L O A D}$ and the reverse current through the freewheeling element.

Neglecting any magnetizing current drawn by the CT, the secondary current $i_{2}$ is related to the primary current $i_{1}$ by

$$
\frac{i_{2}}{i_{1}}=\frac{N_{1}}{N_{2}}
$$

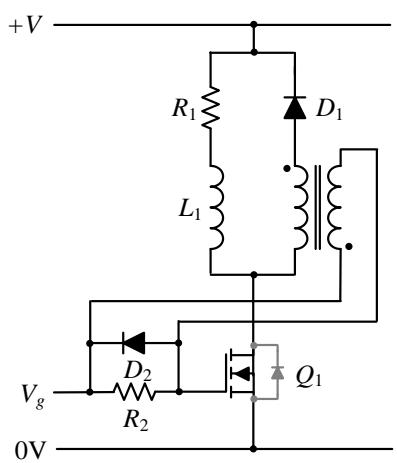

Fig. 9 Circuitry from [15].

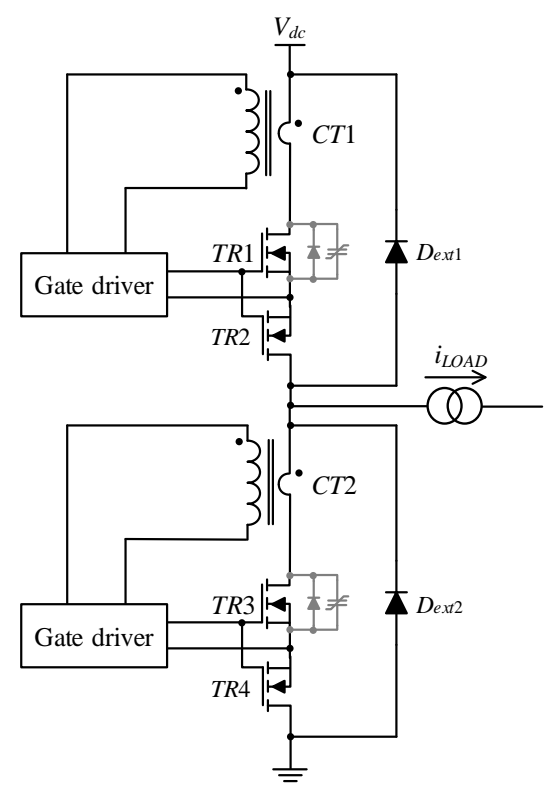

Fig. 10 Bridge-leg equipped with proposed circuitry.

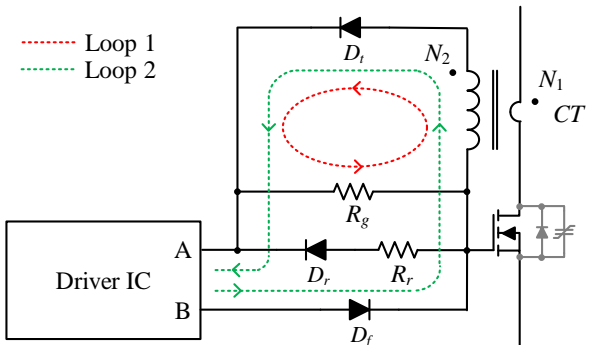

(a)

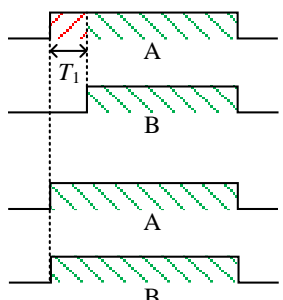

(b)

Fig. 11 (a) Local gate driver circuit. (b) Two output signals, A and B, are produced by the driver IC. In the slow-switching mode a delay time $T_{1}$ is introduced. In the fast-switching mode A and, B are synchronous.

where $N_{1}$ and $N_{2}$ are the CT's primary and secondary turns numbers respectively. If $i_{1}=I_{p k}$ then

$$
i_{2}=\frac{N_{1}}{N_{2}} I_{p k}
$$

At turn-on in the slow-switching mode, only Output A of the driver IC goes high initially, and $i_{2}$ circulates in Loop 1 shown in Fig. 11(a). The voltage $v_{R g}$ across the resistance $R_{g}$ during this interval is given by

$$
v_{R g}=R_{g} i_{2} \text {. }
$$

Putting the result from (14) into (15) gives

$$
v_{R g}=R_{g} \frac{N_{1}}{N_{2}} I_{p k}
$$

To maintain the MOSFET in its pinch-off region, the voltage $v_{R g}$ across $R_{g}$ is approximated by 


$$
v_{R g}=V_{D R I}-V_{G S(t h)}
$$

where $V_{D R I}$ is the high-state output voltage produced by the driver IC in Fig. 11(a) and $V_{G S(t h)}$ is the MOSFET's gate threshold voltage. If this result is put into (16) which is rearranged, then

$$
I_{p k}=\frac{N_{2}\left(V_{D R I}-V_{G S(t h)}\right)}{N_{1} R_{g}} .
$$

After sufficient time is allowed for the turn-on action to elapse, Output B goes high. This clamps the MOSFET's gate to $V_{D R I}$ via a low impedance route. It also allows $i_{2}$ to circulate in the short-circuit loop, Loop 2, for the rest of the MOSFET's on-time. This minimizes the current driven into the CT's magnetizing branch. At turn-on in the fast-switching mode, Outputs A and B rise simultaneously, and the output terminals of the CT are again short-circuited.

When $T R 1$ is off, Outputs A and B are low. When $T R 1$ is off and also acting as the freewheeling device, the charge $Q_{o s s}$ flows through the CT's primary winding when the complementary MOSFET turns on. Again, Outputs A and B present a short-circuit.

\section{EXPERIMENTAL RIG AND RESULTS}

\section{A. Experimental Circuitry}

Key data for the bridge-leg and gate driver circuits are given in Tables I and II. However, in order to evaluate the circuitry, DC tests were carried out using the simplified bridge-leg circuits in Fig. 12.

The CT in Fig. 12(b) was assembled with a TN9/6/3 core in 3F3 material, and with $N_{2}$ formed with 50 turns of $0.2 \mathrm{~mm}$ diameter copper wire. The primary conductor was passed once through the CT's aperture giving $N_{1}=1$. In [19], a CT is used to measure the drain current of a $10 \mathrm{kV} \mathrm{SiC} \mathrm{MOSFET} \mathrm{for}$ over-current monitoring purposes. The CT's primary current in [19] has a low-frequency component, and the core is consequently gapped to avoid saturation. The primary currents in the CTs in Figs. 10, 11, and 12(b) also have a low-frequency component. However, these currents are in the form of a series of return-to-zero pulses, and the core can be reset between pulses. An un-gapped core can therefore be used without saturation occurring. As seen in Figs. 10, 11, and 12(b) the CT is operated without discrete reset circuitry, and reset occurs by means of the resonant action between the CT's magnetizing inductance and parasitic lumped capacitance during the MOSFET's off-times [20]. $R_{g}$ was varied experimentally to set $I_{p k}$, as determined by (18).

The ancillary devices used with $T R 2$ were omitted for these tests as they have little influence on the turn-on behavior

TABLE I. BRIDGE-LEG COMPONENTS FOR CIRCUITRY IN FIG. 10

\begin{tabular}{|c|c|}
\hline$D_{\text {ext } 1,2}$ & SCS220AE \\
\hline$T R 1,3$ & IPW60R041P6 \\
\hline$T R 2,4$ & IRFB7546PBF \\
\hline
\end{tabular}

TABLE II. DRIVER CIRCUIT COMPONENTS FOR CIRCUITRY IN FIG. 11

\begin{tabular}{|c|c|}
\hline$R_{r}$ & $10 \Omega$ \\
\hline$D_{r, f}$ & $1 \mathrm{~N} 4148 \mathrm{TR}$ \\
\hline$D_{t}$ & GB01SLT12-214 \\
\hline Driver IC & IXDN614SI \\
\hline
\end{tabular}

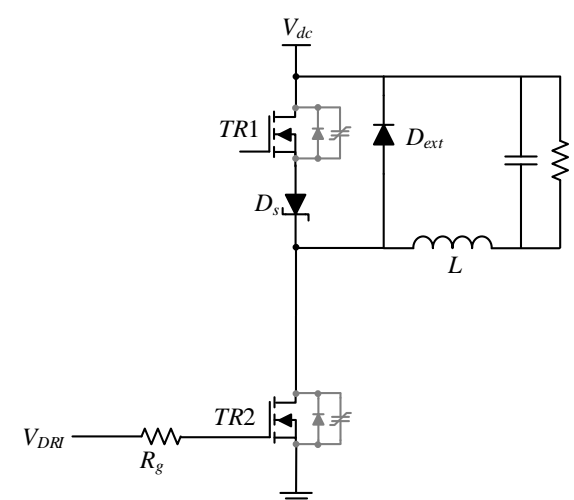

(a)

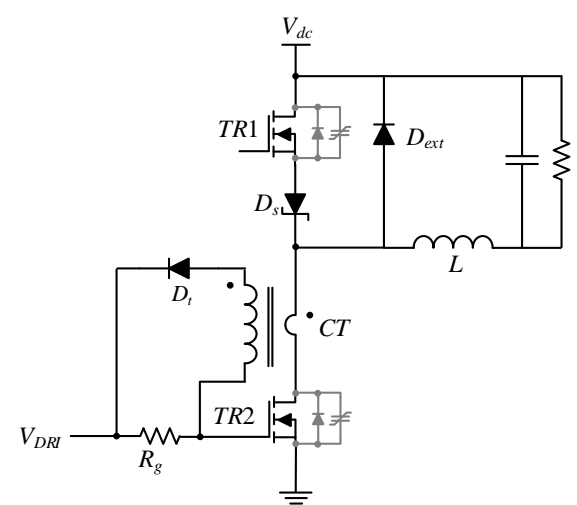

(b)

Fig. 12 Experimental circuits for evaluating methods for controlling incoming drain current profile into MOSFET at turn-on. (a) Simple resistance in series with gate for charging MOSFET input capacitance. (b) Negative feedback from current transformer.

of $T R 2$ in this mode. In each case, $T R 1$ and $T R 2$ were switched in a complementary manner, as occurs in the slow-switching mode. Consequently, the $C_{\text {oss }}$ of $T R 1$ is fully discharged when $T R 2$ is off. For simplicity, a MBR3060CT Schottky diode was used as the anti-series element in these tests instead of a MOSFET as shown in Fig. 10. The supply voltage $V_{d c}$ was $400 \mathrm{~V}$. The gate driver circuits for $T R 1$ and $T R 2$ applied an onstate gate-source voltage $v_{G S}$ of $12 \mathrm{~V}$. The off-state $v_{G S}$ was $-3 \mathrm{~V}$, and it was made negative to prevent $\mathrm{d} v / \mathrm{d} t$-induced simultaneous conduction through $T R 1$ and $T R 2$. The delay time $D$ was defined as the interval between $v_{D R I}$ rising and $v_{D S 2}$ falling to its $50 \%$-level of $200 \mathrm{~V}$.

\section{B. Tests with Simple Resistor in Series with MOSFET'S Gate Terminal}

$R_{g}$ in Fig. 12(a) was set at $160 \Omega, 220 \Omega, 300 \Omega$, and $360 \Omega$. Readings of turn-on energy $E_{\text {on(tri) }}$, peak current $I_{p k}$, and delay time $D$ were taken for $i_{L O A D}=1 \mathrm{~A}, 2 \mathrm{~A}, 3 \mathrm{~A}$, and $4 \mathrm{~A}$.

Fig. 13 shows exemplifying waveforms with $R_{g}=300 \Omega$ and $i_{L O A D}=3 \mathrm{~A}$. $w_{\text {inst }}$ is the instantaneous power dissipation in $T R 2$ and was obtained by multiplying $i_{D 2}$ and $v_{D S 2}$ using the oscilloscope "MATH" function. Fig. 14 shows the relationship between $E_{o n(t r i)}$ and $I_{o s}$ for $I_{L O A D}=1 \mathrm{~A}, 2 \mathrm{~A}, 3 \mathrm{~A}$, and 4 A. Fig. 15 shows the relationship between $E_{o n(t r i)}$ and $I_{p k}$ for $I_{L O A D}=1 \mathrm{~A}, 2 \mathrm{~A}, 3 \mathrm{~A}$, and $4 \mathrm{~A}$. Fig. 16 shows the relationship between $E_{\text {on(tri) }}$ and $D$ for $I_{L O A D}=1 \mathrm{~A}, 2 \mathrm{~A}, 3 \mathrm{~A}$, and $4 \mathrm{~A}$. 


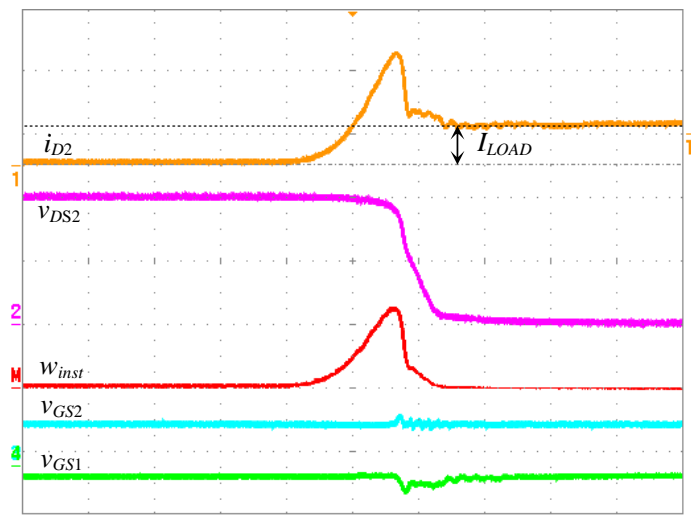

Fig. 13 Exemplifying waveforms from the scheme in Fig. 12(a) with $R_{g}=$ $300 \Omega$ and $I_{L O A D}=3 \mathrm{~A}$. Scales: $i_{D 2}=5 \mathrm{~A} / \mathrm{div} ., v_{D S 2}=200 \mathrm{~V} / \mathrm{div}$., $w_{\text {inst }}=$ $2.5 \mathrm{~kW} / \mathrm{div} ., v_{G S 2}=v_{G S 1}=20 \mathrm{~V} / \mathrm{div}$. Time scale $=200 \mathrm{~ns} / \mathrm{div}$.

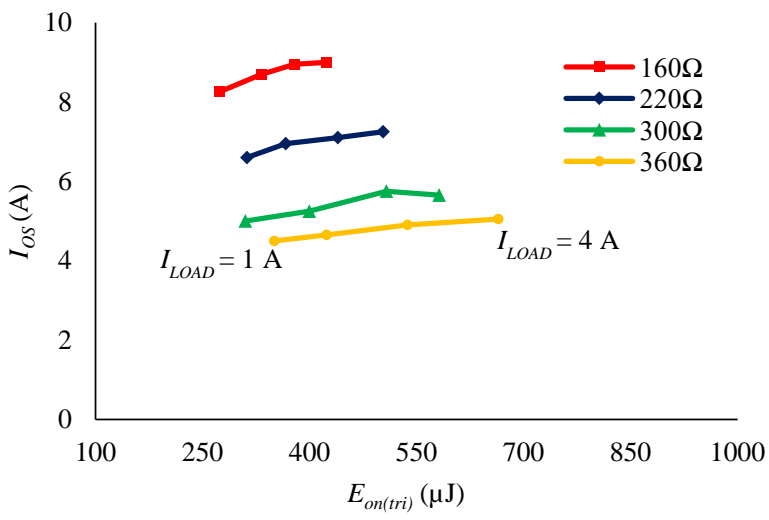

Fig. 14 Relationship between turn-on energy $E_{o n(t r i)}$ and overshoot current $I_{o s}$.

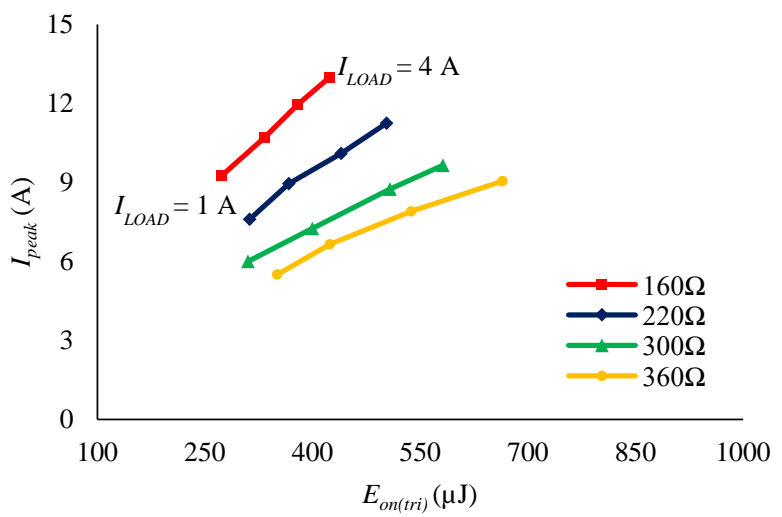

Fig. 15 Relationship between turn-on energy $E_{o n(t r i)}$ and peak current $I_{p k}$.

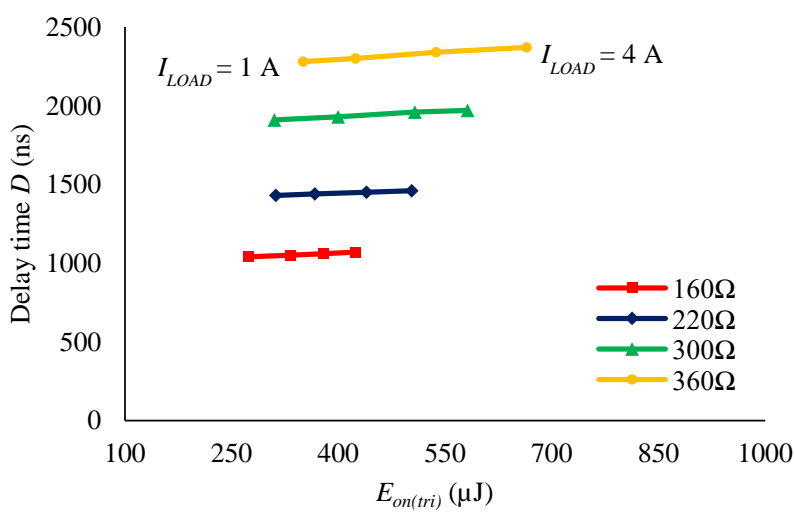

Fig. 16 Relationship between turn-on energy $E_{\text {ontrri }}$ and delay time $D$.

\section{Tests with CT Arrangement}

$R_{g}$ in Fig. 12(b) was set at $39 \Omega, 47 \Omega, 56 \Omega$, and $68 \Omega$. Readings of turn-on energy $E_{\text {on }(\mathrm{rec})}$, peak current $I_{p k}$, and delay time $D$ were taken for $I_{L O A D}=1 \mathrm{~A}, 2 \mathrm{~A}, 3 \mathrm{~A}$, and $4 \mathrm{~A}$.

Fig. 17 shows exemplifying waveforms with $R_{g}=56 \Omega$ and $I_{L O A D}=3 \mathrm{~A}$. Fig. 18 shows the relationship between $E_{\text {on }(\text { rec })}$ and $I_{O S}$ for $I_{L O A D}=1 \mathrm{~A}, 2 \mathrm{~A}, 3 \mathrm{~A}$, and $4 \mathrm{~A}$. Fig. 19 shows the relationship between $E_{o n(r e c)}$ and $I_{p k}$ for $I_{L O A D}=1 \mathrm{~A}, 2 \mathrm{~A}, 3 \mathrm{~A}$, and 4 A. Fig. 20 shows the relationship between $E_{\text {on(rec })}$ and $D$ for $I_{L O A D}=1 \mathrm{~A}, 2 \mathrm{~A}, 3 \mathrm{~A}$, and $4 \mathrm{~A}$

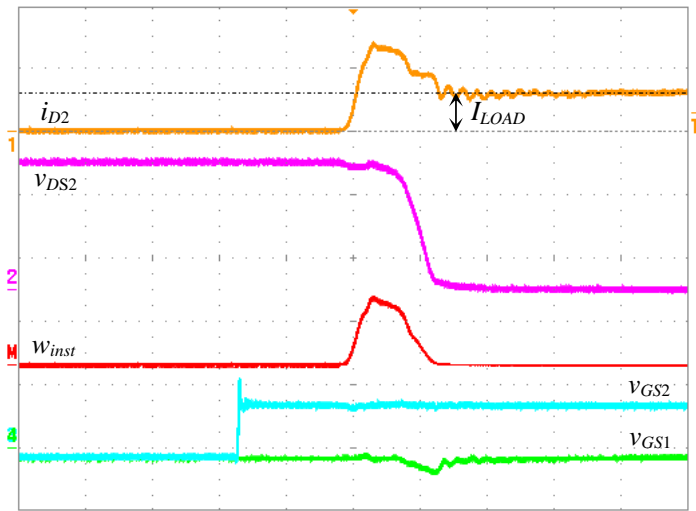

Fig. 17 Exemplifying waveforms from the scheme in Fig. 12(b) with $R_{g}=$ $56 \Omega$ and $I_{L O A D}=3 \mathrm{~A}$. Scales: $i_{D 2}=5 \mathrm{~A} /$ div., $v_{D S 2}=200 \mathrm{~V} /$ div., $w_{\text {inst }}=$ $2.5 \mathrm{~kW} / \mathrm{div} ., v_{G S 2}=v_{G S 1}=20 \mathrm{~V} / \mathrm{div}$. Time scale $=200 \mathrm{~ns} / \mathrm{div}$.

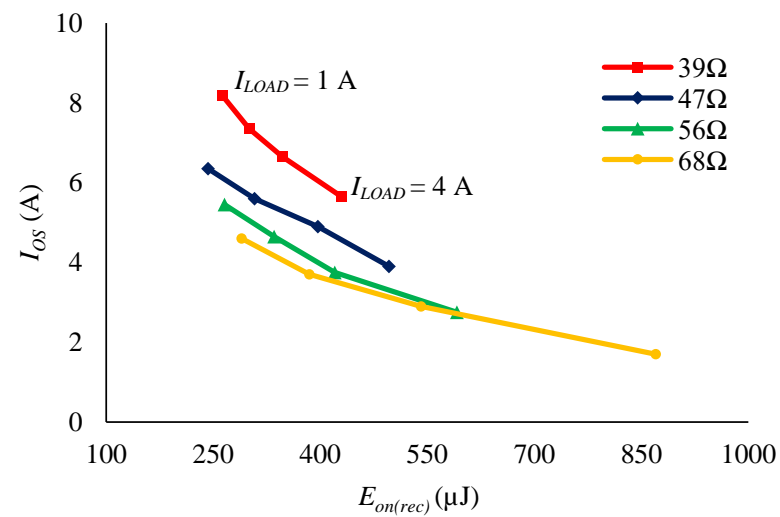

Fig. 18 Relationship between turn-on energy $E_{\text {on (rec) }}$ and overshoot current $I_{o s}$.

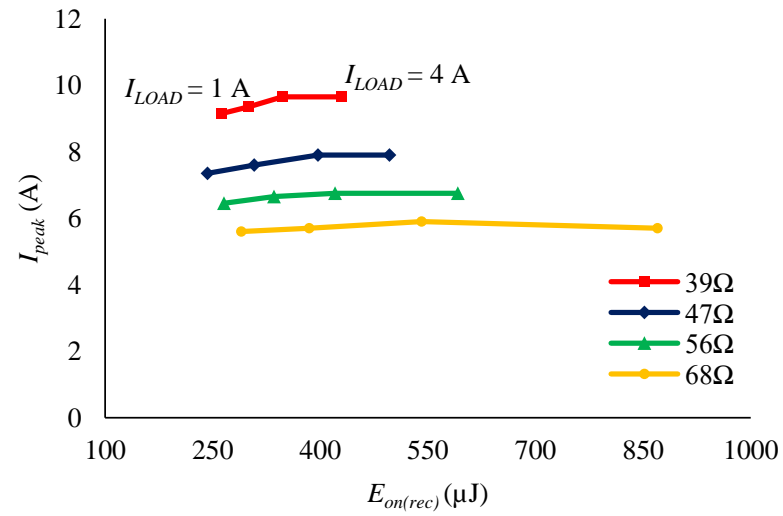

Fig. 19 Relationship between turn-on energy $E_{o n(r e c)}$ and peak current $I_{p k}$. 


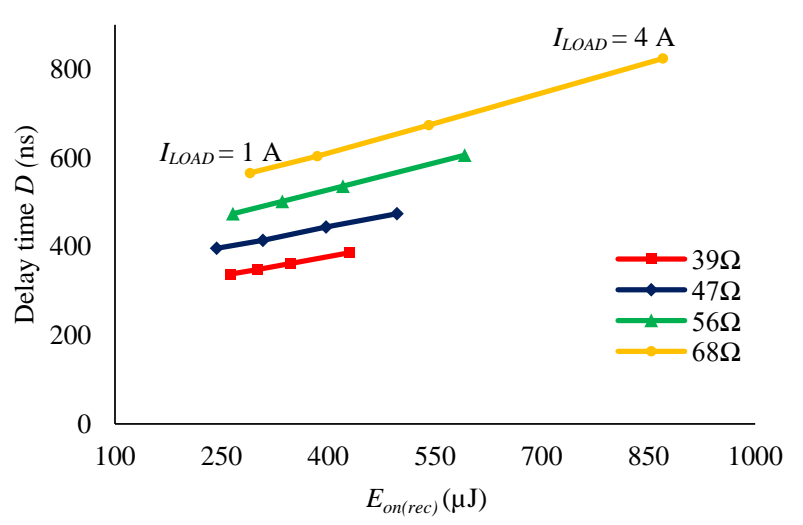

Fig. 20 Relationship between turn-on energy $E_{\text {on(rec })}$ and delay time $D$.

\section{Waveforms from Current Transformer Circuitry}

Exemplifying waveforms from the CT circuitry in Fig. 12(b) were taken with $R_{g}=56 \Omega$ and $I_{L O A D}=3$ A. $i_{2}$ and $v_{2}$ are the CT's secondary current and voltage respectively.

Fig. 21 shows the waveforms when TR2 in Fig. 12(b) turns on. The peak magnetizing current drawn by the CT during the transient turn-on period is very small, and $i_{2}$ is consequently an accurate replica of the primary current $i_{D 2}$ during this period. Fig. 22 shows the waveforms when $T R 2$ in Fig. 12(b) turns off. The half-sinusoidal oscillation of $v_{2}$ is due to the resonant action between the CT's magnetizing inductance and parasitic lumped capacitance during reset. The half-sinusoidal oscillation elapses over a time $t_{r s t}$ which was $816 \mathrm{~ns}$.

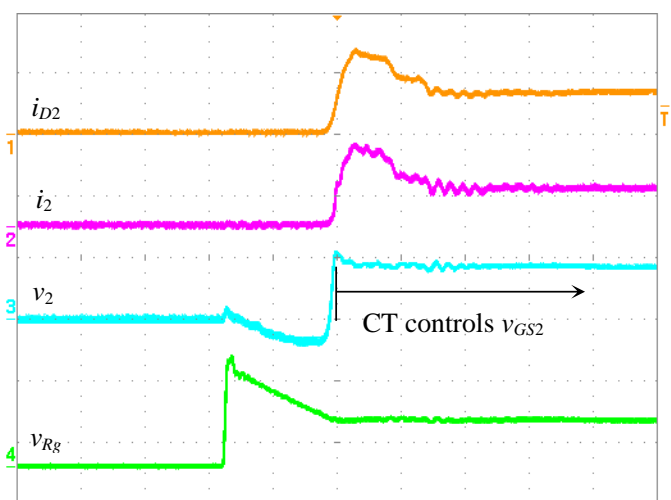

Fig. 21 Exemplifying waveforms from the circuitry in Fig. 12(b) at $T R 2$ turnon with $R_{g}=56 \Omega$ and $I_{L O A D}=3 \mathrm{~A}$. Scales: $i_{D 2}=5 \mathrm{~A} /$ div., $i_{2}=0.1 \mathrm{~A} / \mathrm{div} ., v_{2}$ $=10 \mathrm{~V} /$ div., $v_{R g}=10 \mathrm{~V} /$ div. Time scale $=200 \mathrm{~ns} /$ div.

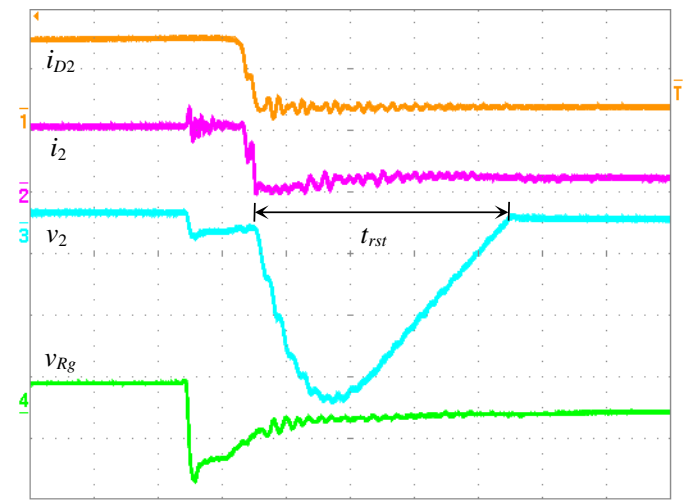

Fig. 22 Exemplifying waveforms from the circuitry in Fig. 12(b) at TR2 turnoff with $R_{g}=56 \Omega$ and $I_{L O A D}=3 \mathrm{~A}$. Scales: $i_{1}=5 \mathrm{~A} /$ div., $i_{2}=0.1 \mathrm{~A} /$ div., $v_{2}=$ $40 \mathrm{~V} /$ div., $v_{R g}=10 \mathrm{~V} /$ div. Time scale $=200 \mathrm{~ns} / \mathrm{div}$.

\section{E. Comparison of Results}

Fig. 23 shows turn-on energies $E_{\text {on(tri) }}$ and $E_{\text {on(rec })}$ plotted against overshoot current $I_{O s}$ at load current $I_{L O A D}=3 \mathrm{~A}$. Fig. 24 shows $E_{\text {on(tri })}$ and $E_{\text {on(rec })}$ plotted against delay time $D$, also at $I_{L O A D}=3 \mathrm{~A}$. In Fig. 23 , under the same $I_{o s}$, the turn-on energy $E_{\text {on(rec })}$ of the rectangular trajectories is less than that of the triangular trajectories $E_{o n(t r i)}$. When $R_{g}$ is made smaller, both $E_{o n(t r i)}$ and $E_{o n(r e c)}$ decrease and $I_{o s}$ increase consequently. In Fig. 24, the $D$ of the rectangular trajectories only has magnitudes of hundreds of nanoseconds, but the $D$ of the triangular trajectories is greater.

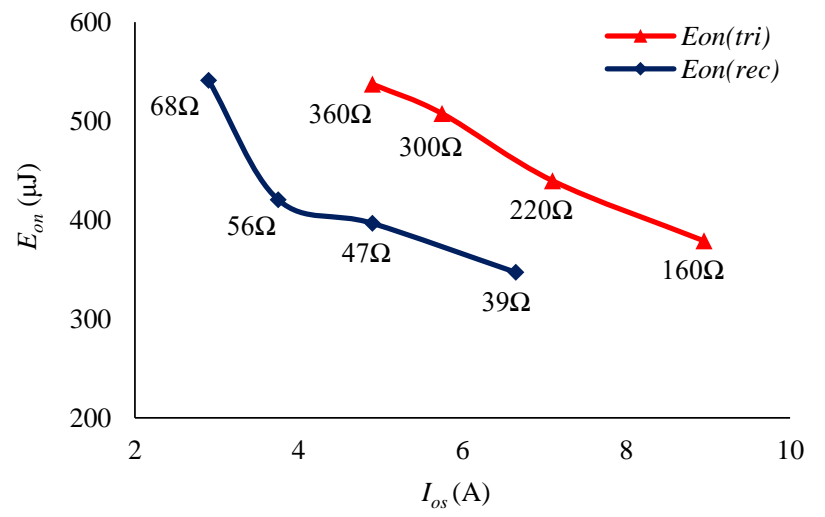

Fig. 23 Turn-on energies $E_{\text {on(tri) }}$ and $E_{\text {on(rec })}$ shown against overshoot current $I_{o s}$ for a load current of $3 \mathrm{~A}$.

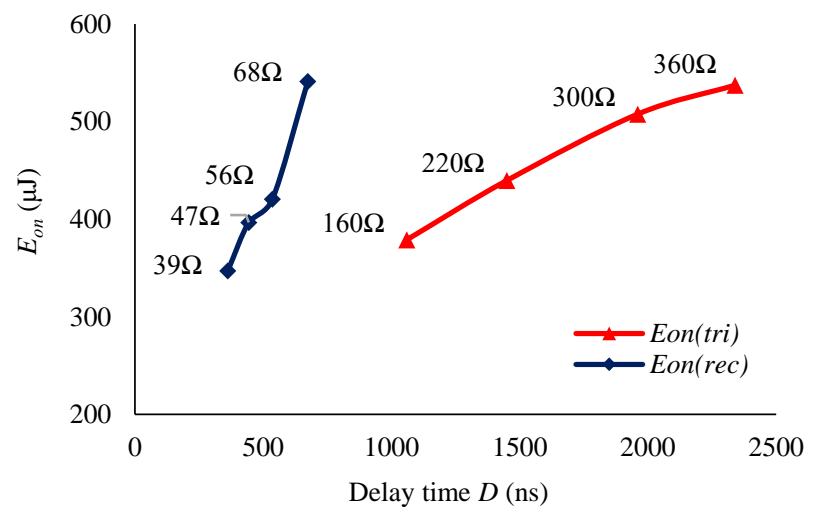

Fig. 24 Turn-on energies $E_{\text {on(tri) }}$ and $E_{\text {on(rec })}$ shown against delay time $D$ for a load current of $3 \mathrm{~A}$.

\section{DISCUSSION}

A conventional gate driver and a gate driver with negative feedback applied to the gate signal by means of a CT have been compared. Improved trade-offs between incoming peak current, turn-on power dissipation, and delay time are obtained with the latter.

Both the conventional and CT circuits are simple, without features such as stepped gate voltage waveforms. The proposed circuitry does not use active gate control [18], [21], and standard gate driver ICs can be used. Whilst the CT is an additional component, it uses a low-cost, compact, and ungapped toroidal core with no safety-isolation requirements.

Whilst the turn-on loss is reduced for a given overshoot current and propagation delay time, it is noted that a loss is incurred by the CT's secondary current flowing through the diodes $D_{t}$ and $D_{f}$ in Fig. 11(a) when the MOSFET is conducting. Both losses have to be evaluated in a practical converter design. 


\section{CONCLUSION}

A gate driver circuit for super-junction MOSFETs in voltage source bridge-legs has been presented. The circuit controls MOSFET incoming drain current profiles. Compared to conventional driver circuits, improved trade-offs between the incoming peak current, turn-on power dissipation, and delay time have been demonstrated. Further investigation will include collecting experimental results when using a bridgeleg in AC tests as in Figs. 10 and 11, and detailed modelling of the switching action of the proposed scheme. This will allow the turn-on trajectories of the MOSFET's drain current and drain-source voltage to be predicted more accurately.

\section{REFERENCES}

[1] J. Rabkowski, D. Peftitsis, and H.-P. Nee, "Silicon carbide power transistors: a new era in power electronics is initiated," IEEE Ind. Electron. Mag., vol. 6, no. 2, pp. 17-26, Jun. 2012.

[2] S. Jahdi et al., "Temperature and switching rate dependence of crosstalk in Si-IGBT and $\mathrm{SiC}$ power modules," IEEE Trans. Ind. Electron., vol. 63, no. 2, pp. 849-863, Feb. 2016.

[3] M. Pfost et al., "Shortcircuit safe operating area of superjunction MOSFETs," in Proc., 28th Int. Symp. Power Semiconductor Devices and ICs, Prague, Czech Republic, Jun. 2016.

[4] L. Lorenz, G. Deboy, and I. Zverev, "Matched pair of Coolmos transistor with SiC-Schottky diode - advantages in application," IEEE Trans. Ind. Appl., vol. 40, no. 5, pp. 1265-1272, Sep. 2004.

[5] F. Udrea, G. Deboy, and T. Fujihira, "Superjunction power devices, history, development, and future prospects," IEEE Trans. Electron Devices, vol. 64, no. 3, pp. 720-734, Mar. 2017.

[6] J. Zhang and J.-S. Lai, "A synchronous rectification featured softswitching inverter using CoolMOS," in Proc., IEEE 21st Annu. Appl. Power Electron. Conf. Expo., Dallas, TX, USA, pp. 810-815, Mar. 2006.

[7] N. McNeill, P. Anthony, and N. Oswald, "Ultra-high efficiency machine drive inverter using super-junction MOSFETs," in Proc., IET 7th Power Electron., Machines Drives Conf., Manchester, UK, Apr. 2014.

[8] A. Hopkins, N. McNeill, and P. H. Mellor, "Drain current injection circuitry for enabling the deployment of super-junction MOSFETs in a 5kW bidirectional DC-DC converter," in Proc., IET 8th Power Electron., Machines Drives Conf., Glasgow, Scotland, Apr. 2016.

[9] A. Hopkins, N. Simpson, and N. McNeill, "Auxiliary resonant source charge extraction circuitry for enabling the use of super junction MOSFETs in high efficiency DC-DC converters," in Proc., IEEE 34th
Appl. Power Electron. Specialist's Conf. Expo., Anaheim, CA, USA, pp. 2159-2166, Mar. 2019.

[10] P. Sun, C. Liu, J.-S. Lai, C.-L. Chen, and N. Kees, "Three-phase dualbuck inverter with unified pulsewidth modulation," IEEE Trans. Power Electron., vol. 27, no. 3, pp. 1159-1167, Mar. 2012.

[11] M. M. Farag, S. M. Gadoue, A. L. Mohamadein, A. M. Massoud, and S. Ahmed, "Elimination of reverse recovery effects associated with Coolmos devices employing current source inverter topology," in Proc., IET 6th Power Electron., Machines Drives Conf., Bristol, UK, Mar. 2012.

[12] J. Kitson and N. McNeill, "A high efficiency voltage-fed quasi Zsource inverter with discontinuous input current using super-junction MOSFETs," in Proc., IET 8th Power Electron., Machines Drives Conf., Glasgow, Scotland, Apr. 2016.

[13] Z. Feng, N. McNeill, and B. W. Williams, "A high-efficiency superjunction MOSFET based inverter-leg configuration using a dual-mode switching technique," in Proc., IEEE 34th Appl. Power Electron. Specialist's Conf. Expo., Anaheim, CA, USA, pp. 2467-2474, Mar. 2019.

[14] D. B. DeWitt, C. D. Brown, and S. M. Robertson, "System and method for reducing body diode conduction," US Patent No. 7508175 (B2), 24 Mar. 2009.

[15] D. Grant, "Diode recovery current suppression circuit," IET Electron. Letts., vol. 38, no. 528, pp. 203-204, 28 Feb. 2002.

[16] S. Mocevic et al., "Comparison and discussion on shortcircuit protections for SiC MOSFET modules: desaturation vs. Rogowski switch-current sensor," IEEE Trans. Ind. Appl., vol. 56, no. 3, pp. 2880-2893, Feb. 2020.

[17] F. Huang and F. Flett, "IGBT fault protection based on $\mathrm{di} / \mathrm{dt}$ feedback control," in Proc., IEEE 38th Power Electron. Specialists Conf., Orlando, FL, USA, pp. 1478-1484, Jun. 2007.

[18] J. Groeger, A. Schindler, B. Wicht, and K. Norling, "Optimized dv/dt, $\mathrm{di} / \mathrm{dt}$ sensing for a digitally controlled slope shaping gate driver," in Proc., IEEE 32nd Appl. Power Electron. Conf. Expo., Tampa, FL, USA, pp. 3654-3569, Mar. 2017.

[19] D. Rothmund, D. Bortis, and J. W. Kolar, "Highly compact isolated gate driver with ultrafast overcurrent protection for $10 \mathrm{kV} \mathrm{SiC}$ MOSFETs," CPSS Trans. Power Electron. Appl., vol. 3, no. 4, pp. 278-291, Dec. 2018.

[20] N. McNeill and N. K. Gupta, "Assessment of the error in the average current sensed by the unidirectional current pulse transformer", IET Circuits Devices Syst., vol. 2, no. 2, pp. 265-276, Apr. 2008.

[21] A. Paredes, H. Ghorbani, V. Sala, E. Fernandez, and L. Romeral, “A new active gate driver for improving the switching performance of $\mathrm{SiC}$ MOSFET," in Proc., IEEE 32nd Appl. Power Electron. Conf. Expo., Tampa, FL, USA, pp. 3557-3563. Mar. 2017. 